\title{
MINIMAL PERTHES' DISEASE
}

\author{
JOHN A. HERRING, MARK A. LUNDEEN, DENNIS R. WENGER
}

From the Texas Scottish Rite Hospital for Crippled Children, Dallas

\begin{abstract}
Of 193 children with Perthes' disease at the Texas Scottish Rite Hospital for Crippled Children, 24 were found to have only minimal changes. The cases were grouped relative to the area of the femoral head involved, with 10 involving the anterior portion, seven the posteromedial, three the lateral, and four the central portion. Almost all hips lost some height as measured by the epiphysial index but all had good results by the Mose criteria irrespective of treatment.

The anterior lesion is the same as that described by Catterall as Group 1. The three additional groups have a similar benign natural history but distinct radiographic features. The need for early recognition of these patterns is emphasised if unnecessary treatment is to be avoided. Possible correlations of these lesions with the segmental blood supply of the femoral head are proposed and an hypothesis relating the Catterall classifications to the blood supply is put forward.
\end{abstract}

Catterall in 1971 delineated four types of involvement of the femoral head in Perthes' disease. His first group was characterised by anterior involvement of the head without collapse or the formation of a sequestrum. His 31 cases of this type resulted in 29 good results and two fair, regardless of treatment. A further report confirmed excellent results without treatment in to inquire into a possible relationship between segmental infarction and segmental blood supply in the femoral head.

MATERIALS AND METHODS

A review was carried out of the radiographs of children with Perthes' disease seen between 1968 and 1977 at the Texas Scottish Rite Hospital for Crippled Children. Criteria for selection included partial involvement of the femoral head with no sequestrum formation nor

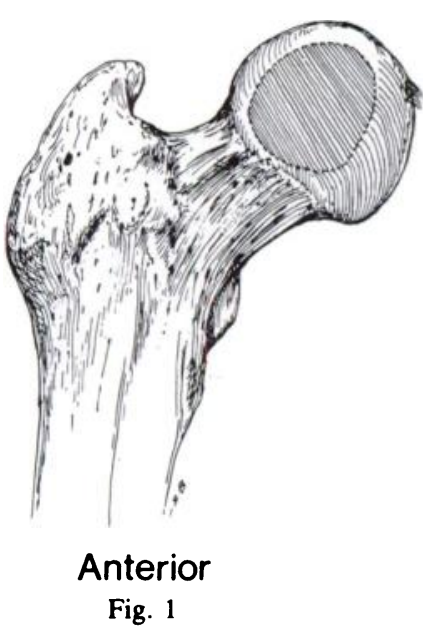

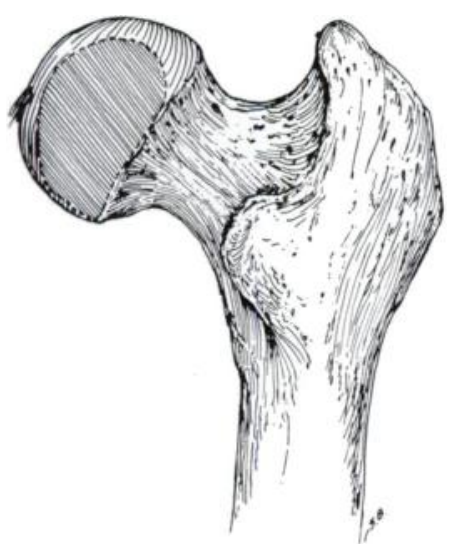

Posteromedial

Fig. 2

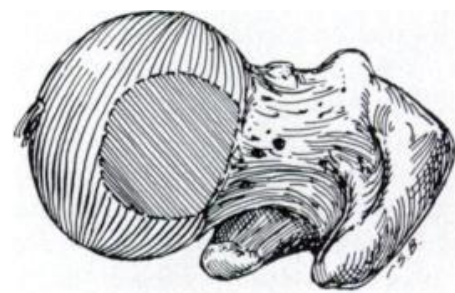

Lateral

Fig. 3

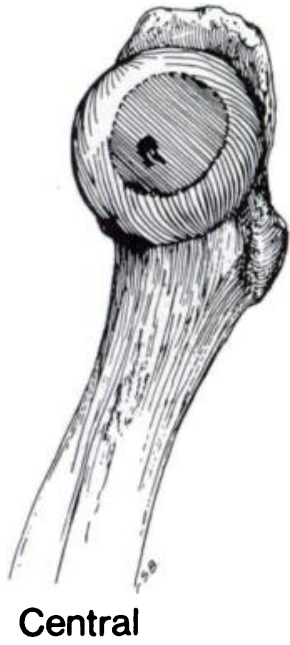

Fig. 4

Drawings of the four typical lesions as seen from the anterior, posterior, superior and medial views respectively.

patients of Catterall Group 1 (Lloyd-Roberts, Catterall and Salamon 1976).

We have noted significant variations in the less severe form of Perthes' disease not reported by Catterall, and this study was undertaken to define these variations, to discover the duration of mild disease, and collapse, and sequential density changes which followed the usual Perthes' pattern.

We initially used the Catterall criterion of anterior involvement; however, we found a significant number of cases with minimal involvement of other areas of the femoral head which thus did not fit Catterall's description of Group 1. We found the patterns to be distinct enough to be classified according to area of involvement (Figs 1 to 4). 


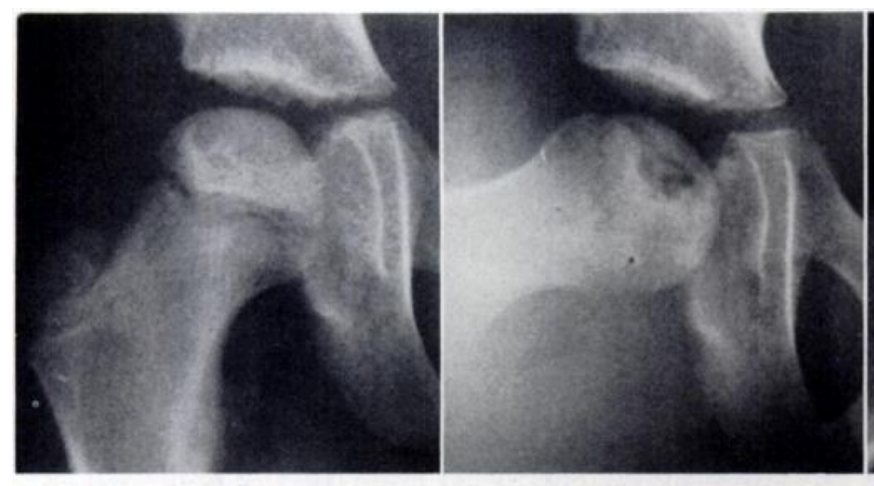

Fig. 5
Fig. 6

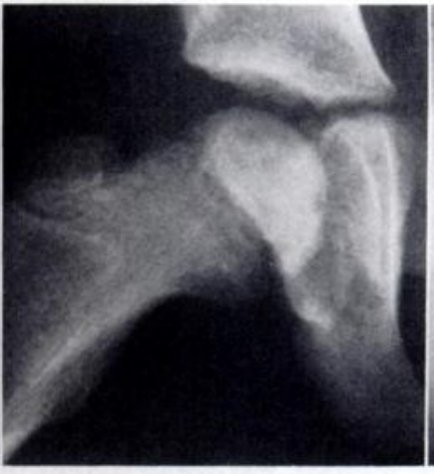

Fig. 7

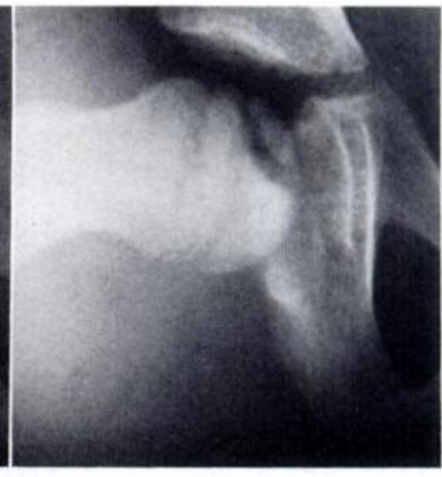

Fig. 8

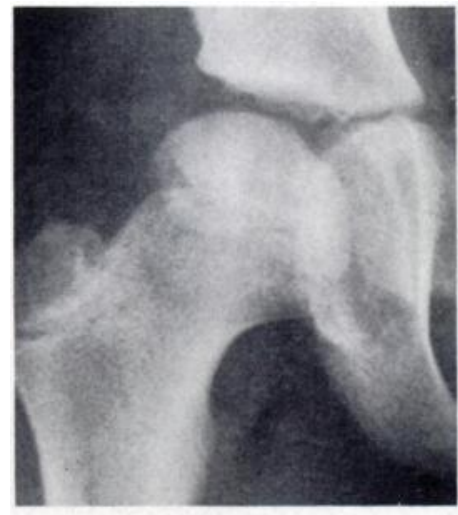

Fig. 9

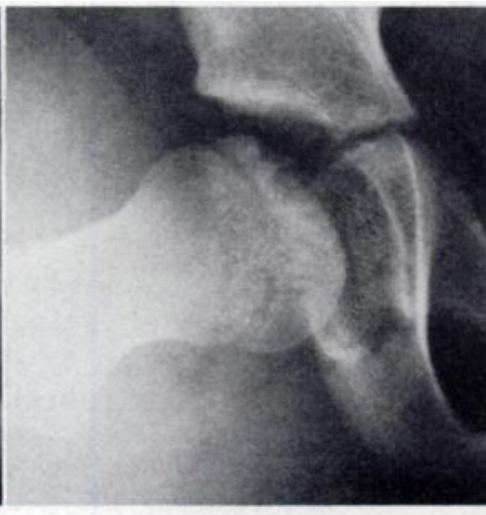

Fig. 10

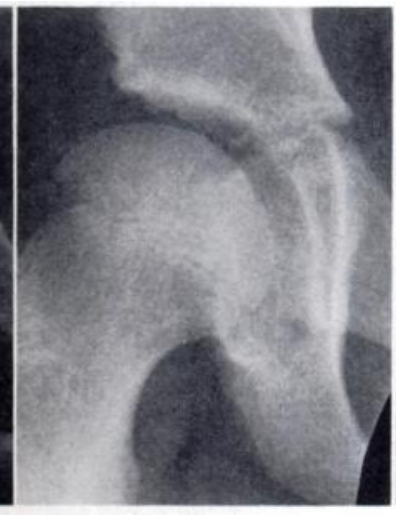

Fig. 11

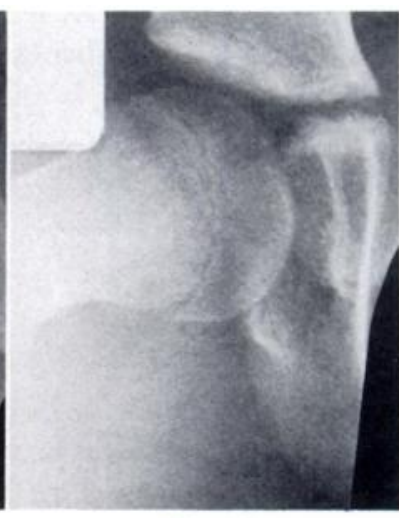

Fig. 12

Case 1. Anterior lesion in a boy aged eight and a half years. Initial radiographs: Anteroposterior radiograph (Fig. 5) showing central density changes, lateral radiograph (Fig. 6) with anterior metaphysial lucency. Four months afier onset: More diffuse central changes (Fig. 7) and well-demarcated lesion of the anterior segment (Fig. 8). Twenty months after onset: Central lucent area and incomplete reossification (Fig. 9) and healing of the anterior lesion (Fig. 10). Thirty-six months after onset: Complete reossification and no loss of sphericity (Figs 11 and 12).

One hundred and ninety-three cases were reviewed. From this group 24 hips in 24 patients met the criteria. There were 23 White children and one Black, 22 boys and two girls. Fifteen patients had Catterall Group 2, 3, or 4 involvement of the contralateral hip (11 of these hips received treatment) and the minimally involved hip was an incidental finding. Adequate anteroposterior and lateral radiographs were available on all hips throughout the course of the disease.

Grouping of cases. The 24 hips were grouped according to area of involvement of the femoral head.

Anterior involvement (10 hips). In this group the density changes on the anteroposterior radiograph were limited to the laterocentral portion of the femoral head, leaving the extreme lateral portion intact. On the lateral radiograph only the anterior portion of the head was involved. This group corresponds to Catterall's Group 1 (Fig. 1).

Posteromedial involvement (seven hips). In this group the anteroposterior radiograph showed changes in the medial and central area. On the lateral view the changes were in the posterior half of the head (Fig. 2).

Lateral involvement (three hips). In this group avascular changes were limited to the lateral portion of the head on the anteroposterior radiograph. On the lateral view the changes were centrally located and the anterior portion was not involved (Fig. 3).

Central involvement (four hips). In this group avascular changes appeared centrally located on both the anteroposterior and lateral views (Fig. 4).

In addition to the four cases of minimal disease with central involvement, four additional cases were reviewed with superficial cystic changes in the central portion of the femoral head. These changes resolved completely over a period of time ranging from $\mathbf{2 0}$ to 33 months. The significance of this finding is unclear. There was insufficient evidence to confirm a diagnosis of Perthes' disease and these hips are not included in the study. All these patients had Perthes' disease of the contralateral hip and all were between four and five years of age.

\section{RESULTS}

Age. Age at the time of diagnosis ranged from two years ten months to nine years nine months and averaged five years six months. There was no significant difference in ages among the four groups.

Race and sex. The one Black patient had lateral involvement. The two girls had posteromedial involvement.

Signs and symptoms. Thirteen left hips and 11 right hips were involved. Fifteen patients had symptoms before evaluation ranging in duration from one week to 12 months with an average of four months. Nine patients were asymptomatic. Many patients had some guarding at the extremes of movement but none had significant restriction of movement of the hip.

Treatment. Sixteen patients were treated by traction followed by an abduction splint attached to the feet, with the feet medially rotated, and a bar and cuff attached above the knees to hold the hip in abduction and medial rotation. The period of treatment ranged from 3 to 18 months with a mean duration of 12 months. The children could walk in this splint. Eight patients had no treatment. 


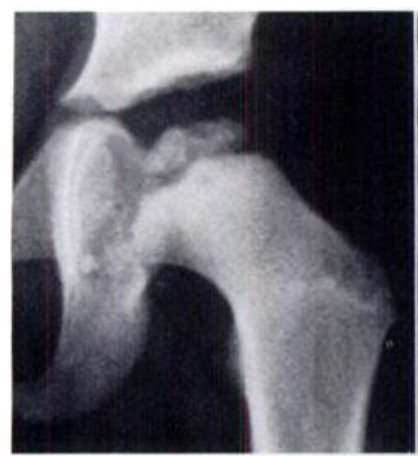

Fig. 13

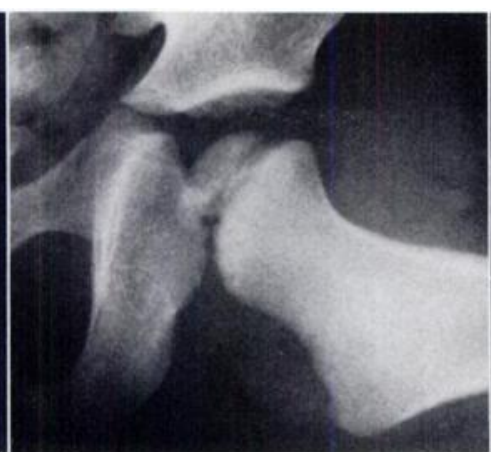

Fig. 14

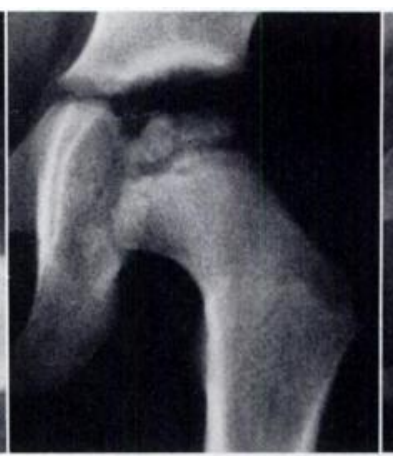

Fig. 15

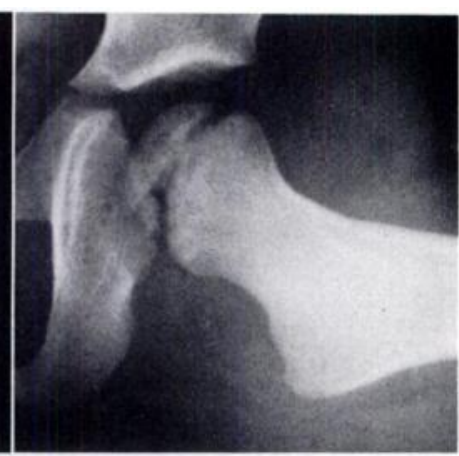

Fig. 16

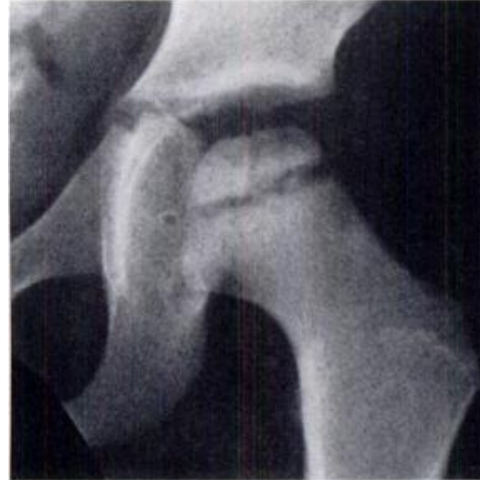

Fig. 17

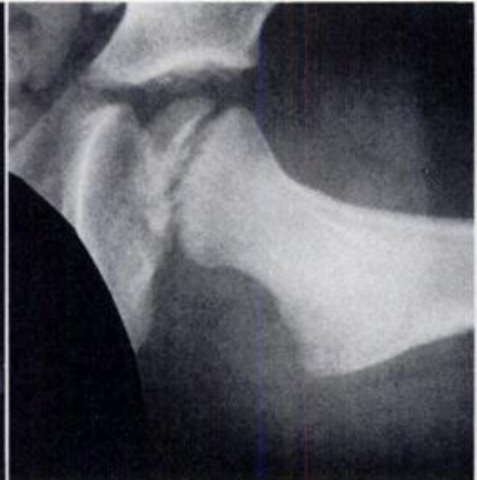

Fig. 18

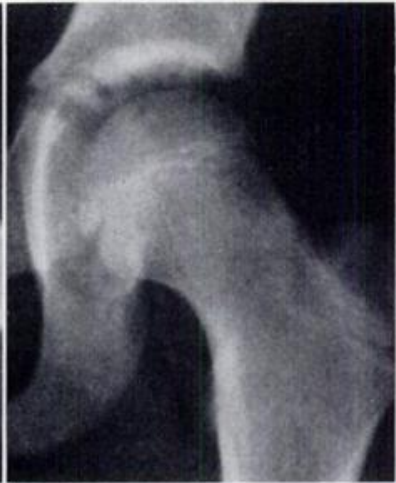

Fig. 19

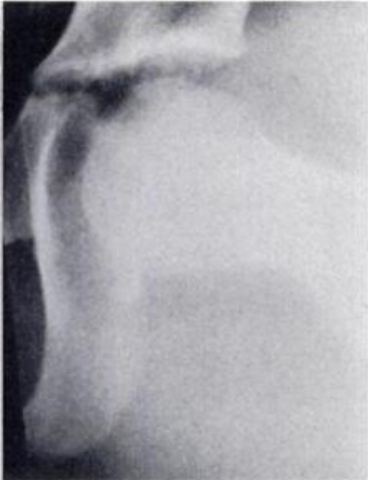

Fig. 20

Case 2. Posteromedial lesion in a child aged two years ten months. Initial radiographs: Anteroposterior radiograph (Fig. 13) showing mottled density changes throughout the femoral head. Lateral radiograph (Fig. 14) showing increased density to posterior segment of the femoral head. Ten months after onset: Demarcation between the denser medial portion of the femoral head and the less dense lateral portion (Fig. 15), and fragmentation of the posterior portion of the femoral head (Fig. 16). Sixteen months after onset: The medial segment still has increased density (Fig. 17) and there is persistent fragmentation of the posterior portion of the femoral head (Fig. 18). Eight years after onset: Complete reossification of the femoral head and no loss of sphericity (Figs 19 and 20).

Radiographic review. The epiphysial index of the initial and final radiographs was determined and the difference was measured. In all but two cases there was a decrease in the epiphysial index over the course of the disease. This ranged from a difference of -0.01 to -0.19 and averaged -0.06 . There was no significant difference between the groups in regard to the amount of change in the epiphysial index. When evaluated by the Mose (1964) criteria all results were classified as good. Neither the epiphysial index nor the Mose template showed differences between the hips which had been treated and those which had not.

The location of metaphysial radiolucency was found to correlate with the location of femoral head involvement in 12 hips (see Fig. 29). In the anterior group there were two hips with diffuse metaphysial changes, two hips with central metaphysial changes, and six hips with anterior metaphysial changes. In the posteromedial group one hip had posteromedial metaphysial changes and six had no metaphysial changes. In the central group two hips had central metaphysial changes and two hips had no metaphysial changes.

The distance from teardrop to metaphysis was mildly increased in all groups. The measurement from the outer wall of the teardrop to the metaphysis ranged from 5 to 14 millimetres (average 10.2 millimetres) and was not significantly different between the groups.

The duration of the disease was calculated in seven hips in which the first film appeared normal and in which subsequent changes of Perthes' disease were noted. Completion of the disease was defined as the time at which all density changes ceased to evolve and either a minimal dense scar or no changes remained in the femoral head. In these hips the duration of the disease ranged from 17 to 36 months with an average of 21.3 months.

All hips were reviewed to determine the earliest time at which the diagnosis of minimal involvement of the head could be established. This ranged from zero months (minimal involvement evident on the first film) to 20 months and averaged five months.

The degree of involvement and the outcome of the contralateral hips were evaluated using the Mose criteria. Ten hips with Catterall Group 2 involvement had nine good results and one fair; three hips with Catterall Group 3 involvement had two fair results and one poor; and two hips with Catterall Group 4 involvement had one fair and one poor result.

Four cases are illustrated in Figures 5 to 32 . 


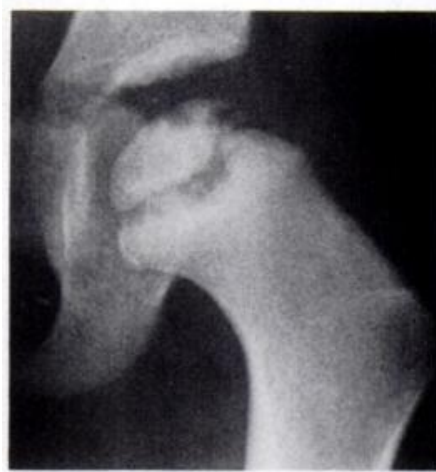

Fig. 21

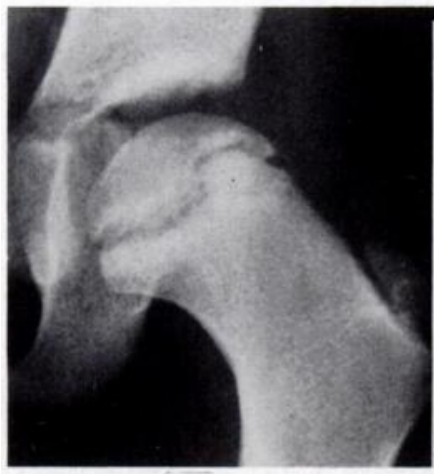

Fig. 25

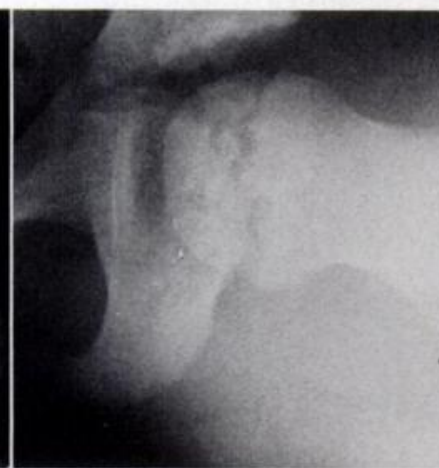

Fig. 22

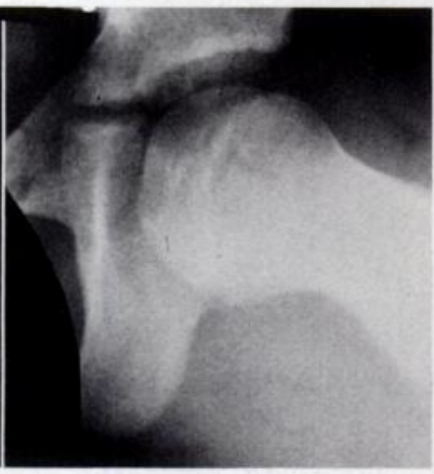

Fig. 26

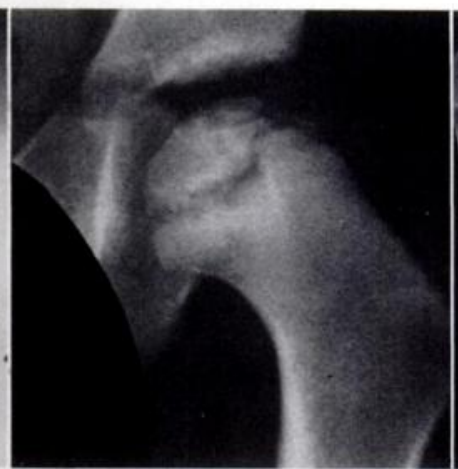

Fig. 23

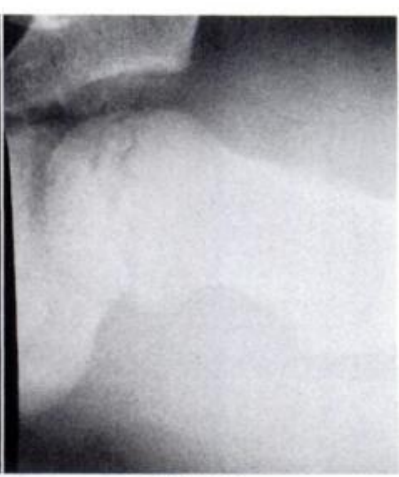

Fig. 24

Case 3. Lateral lesion in a boy aged five years four months. Initial radiographs: Anteroposterior radiograph (Fig. 21) showing lucency in the lateral portion of femoral head. Lateral radiograph (Fig. 22) showing no abnormalities. Five months after onset: Continued fragmentation laterally (Figs 23 and 24). Forty months after onset: Healing of the lesion and no loss of sphericity (Figs 25 and 26).

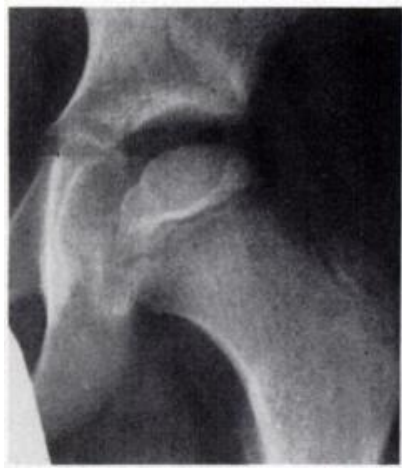

Fig. 27

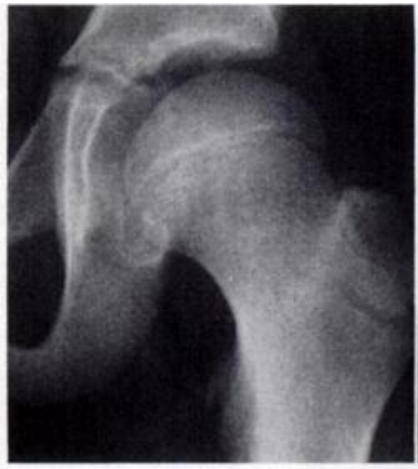

Fig. 31

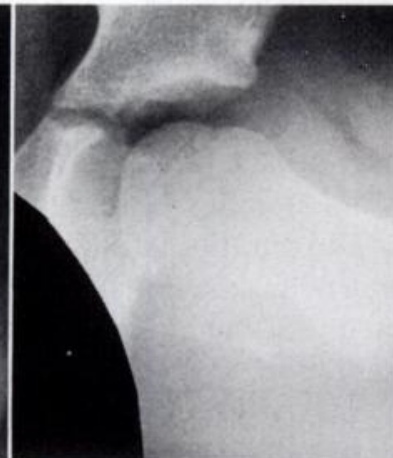

Fig. 28

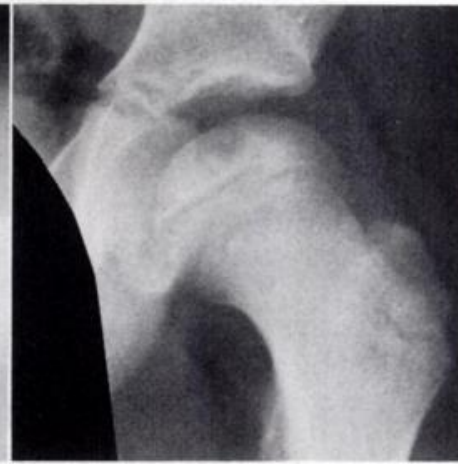

Fig. 29

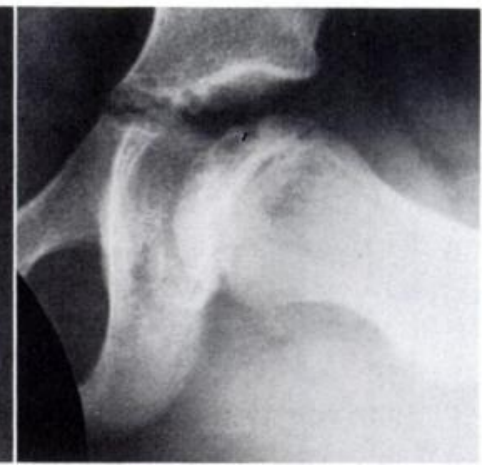

Fig. 30

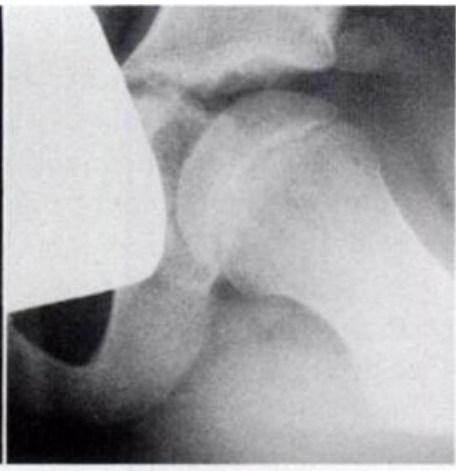

Fig. 32

Case 4. Central lesion in a boy aged eight years four months. Initial radiographs: Anteroposterior radiograph (Fig. 27) showing central density changes, and lateral radiograph (Fig. 28) with no distinct changes. Fourteen months after onset: Small area of central lucency (Fig. 29) and central area of fragmentation (Fig. 30). Anterior portion of the head is intact. Thirty-five months afier onset: Complete reossification (Figs 31 and 32). There has been no loss of sphericity. 


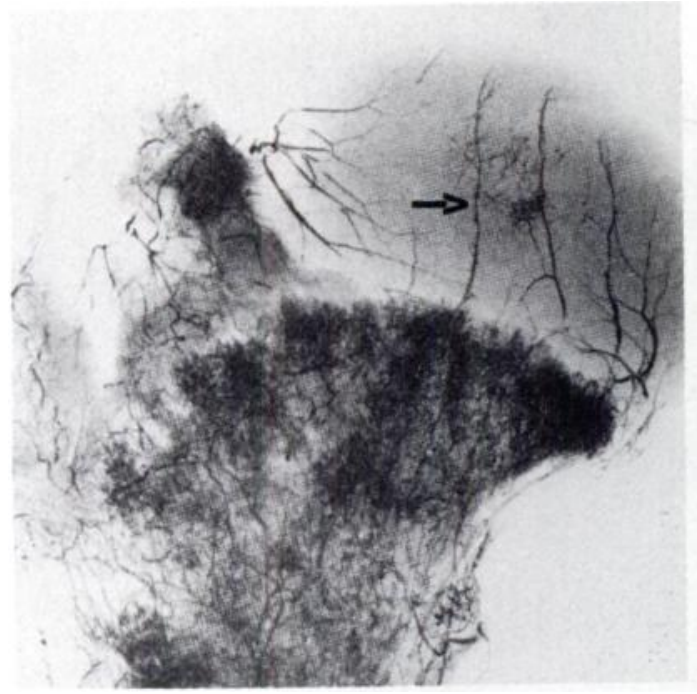

Fig. 33

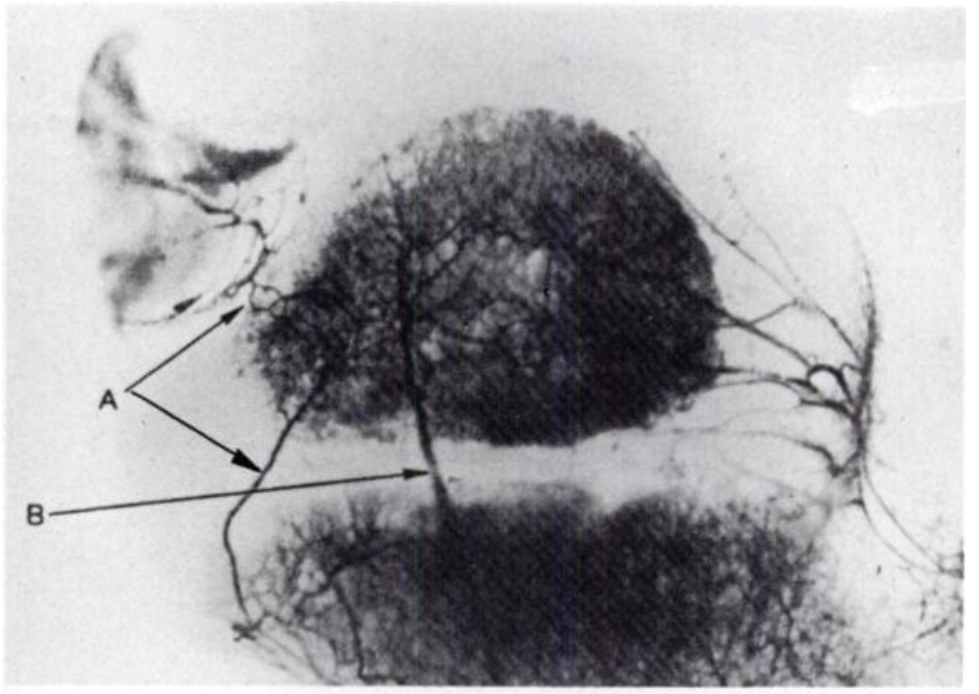

Fig. 34

Figure 33-Anterior blood supply (from Trueta). Anterior ascending cervical arteries (arrow) supply the anterior portion of the femoral head. Figure 34-Posteromedial blood supply (after Chung). Medial $(A)$ and posterior $(B)$ ascending cervical arteries to the posteromedial portion of the femoral head.

\section{DISCUSSION}

In this series of 24 hips with minimal Perthes' disease, four patterns of involvement of the femoral head were found. The diagnosis was appropriate because of the characteristic evolution of changes in the density of the femoral head and metaphysis, the age distribution, and the frequent occurrence of more typical Perthes' disease in the contralateral hip. The group with anterior involvement is identical to the Catterall Group 1, but the other three groups have not previously been described. Because of the benign natural history of all four groups, we propose that they be considered as subgroups of Catterall Group 1.

While 15 patients had mild symptoms, none had significant loss of movement. Thus the diagnosis of minimal Perthes' disease should be considered when patients present with a full range of movement. In most cases the narrow localisation of radiographic changes was evident within six months of the onset of symptoms. The duration of radiographic evidence of disease was similar to the more usual forms of Perthes' disease and averaged 21.3 months. The results were uniformly good whether treatment was received or not and therefore treatment seems unnecessary. Thus, recognition of these patterns is most important so that containment treatment may either be avoided altogether or discontinued if started while awaiting specific diagnosis.

It is generally accepted that Perthes' disease is the result of a temporary, perhaps recurrent, loss of blood supply to the femoral head. Many elegant studies of the vasculature of the femoral head have been performed seeking to explain the disease and its genesis (Trueta 1957; Wertheimer and Lopes 1971; Lauritzen 1974; Ogden 1974; Chung 1976). Chung has suggested that anterior deficiency of the extracapsular arterial ring could render the bone supplied by the lateral ascending cervical arteries more vulnerable to necrosis when flow is impeded posteriorly. He found the secondary anastomoses of the subsynovial ring to be frequently deficient, especially in boys, and proposed that this related to the male predominance of the disorder.

We have carefully reviewed the vascular patterns of the femoral head and have found remarkable similarities between these patterns and the patterns of necrosis reported in this paper. Perhaps a study of these limited lesions will demonstrate patterns which are obscured when more massive necrosis occurs. Consideration will be given to each of the four patterns of involvement relative to the usual blood supply of that segment.

The anterior portion of the femoral head is supplied by one or two anterior ascending cervical arteries derived from the lateral femoral circumflex artery. Penetrating branches of these vessels supply the anterior metaphysis (Fig. 33). The anterior lesion may result from occlusion or malformation of these vessels. The anterior metaphysial changes may be mediated through the penetrating metaphysial branches of these vessels.

The posteromedial portion of the head is supplied by medial and posterior ascending cervical arteries which, if occluded or injured, would produce a posteromedial lesion. The viability of the remainder of the head would be based on the intact lateral ascending vessel (Fig. 34). Again, there are penetrating branches of these arteries in the area of the metaphysis where we find radiographic changes.

The lateral portion of the femoral head is supplied by branches of the lateral ascending cervical vessel. In most hips this vessel provides the major part of the vascular network of the femoral head (Fig. 35). The lateral lesion may be due to injury, occlusion, or malformation of the small lateral branches without involvement of the main trunk of the artery. 


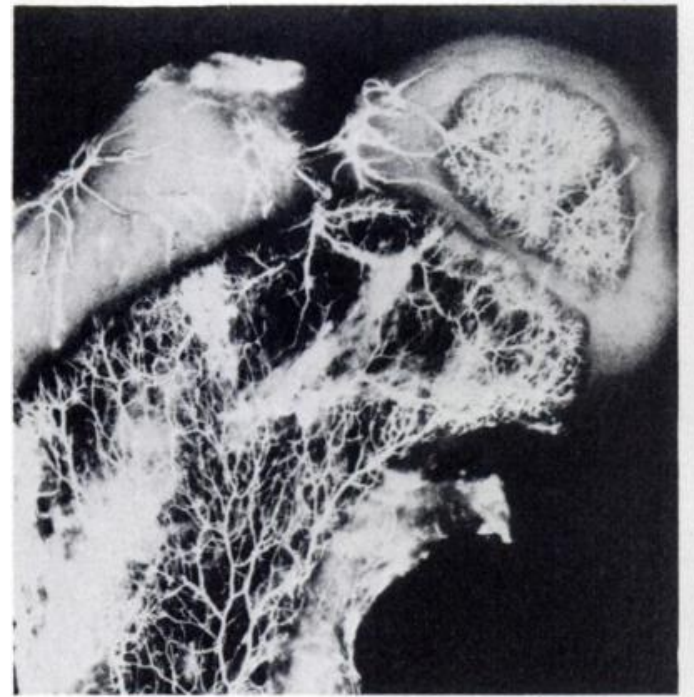

Fig. 35

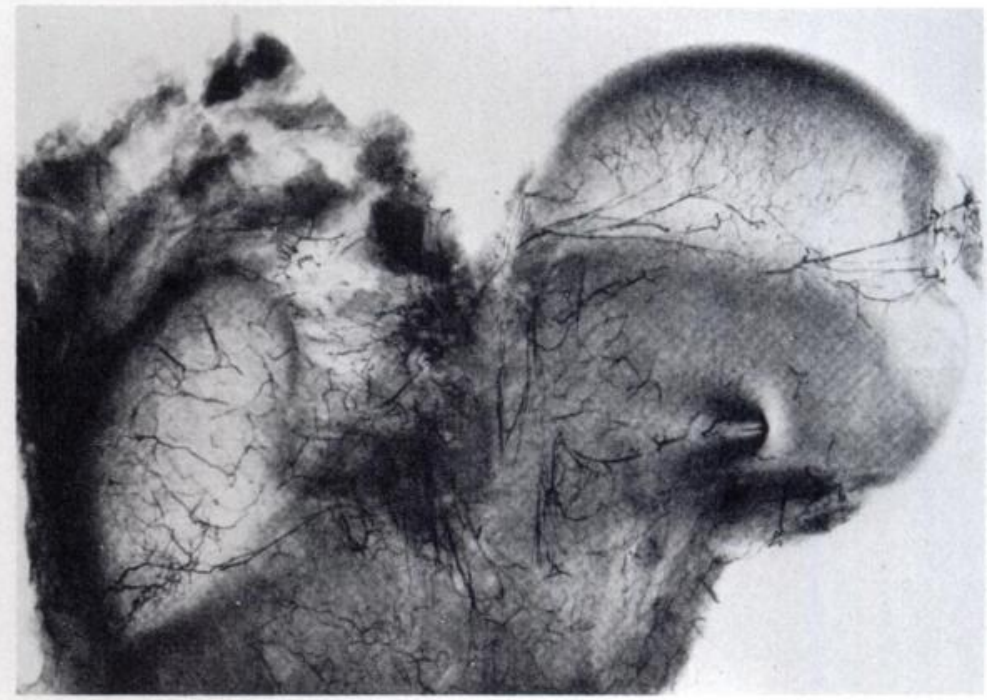

Fig. 36

Figure 35-Lateral blood supply (from Trueta). Lateral ascending cervical artery with peripheral branches to the lateral portion of the femoral head. Figure 36-Central blood supply (from Trueta). Central femoral head receiving its vascular supply from the vessels of the ligamentum teres.

There are at least three possible sources of blood supply to the central portion of the femoral head. This area is most commonly supplied by the terminal portion of the lateral ascending artery (Fig. 35). Occasionally, supply is from medial or posterior vessels (Fig. 34) and rarely by the vessels of the ligamentum teres (Fig. 36). Thus, the central lesion might have its origin in one of three different vascular systems.

Finally, a similar hypothesis can be applied to the more severe forms of Perthes' disease. Perhaps the Catterall Group 2 form of the disease corresponds to an infarct of the lateral ascending cervical vessel distal to the origin of the branches to the lateral portion of the head. The viable medial pillar might be supplied by intact medial vessels (Fig. 37). The Catterall Group 3 lesion may represent infarction of the lateral vessel proximal to the lateral branches with medial viability again based on the medial ascending cervical vessels (Fig. 37). Finally, the Catterall Group 4 lesion may be due to a lateral ascending anterior infarct in a head with little other source of supply (Fig. 35) as is often the case.

Future studies which may confirm or refute these

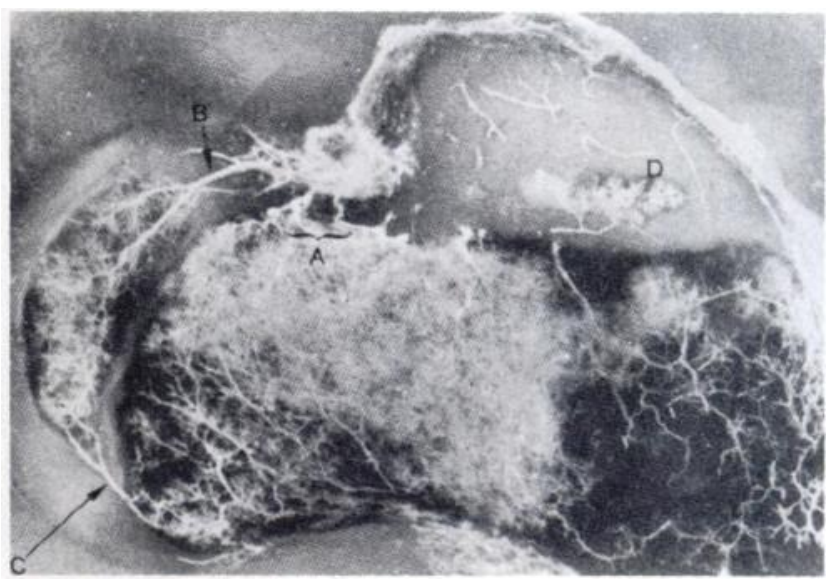

Fig. 37

Lateral ascending cervical artery $(B)$ and medial ascending cervical artery $(C)$ supplying central femoral head (from Chung). Occlusion at $(B)$ could produce a Catterall Group 2 lesion; at $A$ a Catterall Group 3 lesion.

hypotheses should include high-resolution bone scanning, experimental animal studies, and close scrutiny of other clinical material.

Acknowledgement is given to the Journal of Bone and Joint Surgery for Figures 34 and 37 which are reproduced by permission of the American Volume, and for Figures 33, 35 and 36 which are reproduced by permission of the British Volume.

\section{REFERENCES}

Catterall A. The natural history of Perthes' disease. J Bone Joint Surg [Br] 1971;53-B:37-53.

Chung SMK. The arterial supply of the developing proximal end of the human femur. J Bone Joint Surg [Am] 1976;58-A:961-70.

Laurtizen J. The arterial supply to the femoral head in children. Acta Orthop Scand 1974;45:724-36.

Lloyd-Roberts GC, Catterall A, Salamon PB. A controlled study of the indications for and the results of femoral osteotomy in Perthes' disease. $J$ Bone Joint Surg [Br] 1976;58-B:31-6.

Mose K. Legg-Calvé-Perthes disease. Universitetsforlaget I. Aarhus, 1964.

Ogden JA. Changing patterns of proximal femoral vascularity. J Bone Joint Surg [Am] 1974;56-A:941-50.

Trueta J. The normal vascular anatomy of the human femoral head during growth. $J$ Bone Joint Surg [Br] 1957;39-B:358-94.

Wertheimer LG, Lopes SDe LF. Arterial supply of the femoral head: a combined angiographic and histological study. J Bone Joint Surg [Am] $1971 ; 53-A: 545-56$. 